\title{
IoT Based Human Activity Recognition System Using Smart Sensors
}

\author{
Deepti Sehrawat*, Nasib Singh Gill \\ Department of Computer Science \& Applications, Maharshi Dayanand University, Rohtak, Haryana, 124001, India
}

\begin{tabular}{l} 
A R T I C L E I N F O \\
\hline Article history: \\
Received: 24 May, 2020 \\
Accepted: 19 July, 2020 \\
Online: 09 August, 2020 \\
\hline
\end{tabular}

Keywords:

Types of IoT Sensors

HAR Systems

Sensor Applications

IoT and Sensors

\begin{abstract}
A B S T R A C T
Internet of Things provides a virtual view of real-life things by guiding challenges faced by persons in daily life. It is reforming our world with trillions of sensors and other IoT enabled devices by creating a smart environment. Effective use of IoT sensors in various smart IoT applications is very important. After analyzing different sensor applications, this paper presents various types of wearable sensors used for monitoring of human activities along with different locations optimal for their placement. This paper enlightens sensors suitable for any particular application. IoT has opened up a new avenue of research in the field of human activity recognition using wearable sensors. It provides an individual's valuable information regarding functional ability and lifestyle. Human activities are monitored automatically to provide personalized support to different individuals. Recently, various researchers presented different human activity recognition systems, a few are cumulated in this paper. Furthermore, a Human Activity Recognition (HAR) system is also proposed in this paper for a smart IoT environment that would be secure enough to use.
\end{abstract}

\section{Introduction}

The advent of Internet of Things (IoT) has opened a plethora of opportunities. In recent years, human activity recognition has received growing attention. This paper is an extension of work originally presented in the 2019 3rd International Conference on Trends in Electronics and Informatics (ICOEI) [1]. IoT not only connects animate/living things but inanimate/non-living things are also connected in a smart environment. This brings revolutionary changes in the world [2]. In any IoT based applications, sensors are very significant. Any physical/chemical change can be detected by these sensors which are then collected and processed to automate the application/devices to make it smarter in the form of smart application. IoT integrates several sensors of different types attached to the devices and nodes that can communicate without any human intervention [3].

Wearable sensors and the advances of wireless networks has opened the door to a world of novel interactive IoT applications. Different types of inexpensive and unobtrusive body-worn sensors like accelerometers, gyroscopes, and microphones have been often used in daily life activities [4]. Various researchers worked on detecting human activities by observing different parameters. It can be used for the monitoring of people of any age group like for infants, young people, disabled people, and elderly people in a

*Corresponding Author: Deepti Sehrawat, dips.scorpio@gmail.com solitary life. These monitoring systems are usually smaller and are wearable [5].

The mental or physical health of a user can be monitored remotely and then the results can be sent to their remote family. Most of the earlier researchers in this area made use of multiple body-worn sensors worn/ placed at different body parts like chest, thigh, waist, ankle, knee, wrist, ear, eyes, and so on [6]. The purpose of those research work was to categorize a definite subset of activities for particular applications. For instance, for upper body movements, wrist and arm sensors were used whereas for simple lifestyle monitoring a single sensor would be enough [7]. Most of the human activity recognition systems use accelerometers for the classification and assessment of day-to-day activities. Some used single accelerometers while some used multiple accelerometers attached to different sites on one's body. Wearing multiple accelerometers gives a good level of accuracy for certain activities but wearing multiple sensors all the time is not feasible. Wearable sensors are mostly used to detect physical movements but these can also be used for monitoring academic performance, analysing sleep class/patterns, stress level, and mental fitness conditions. Researchers in [8] gathered data from 66 individuals and analysed behavioural and physiological patterns. They also identified factors affecting performance in academics, sleep quality, stress level, and mental health. In the process of human activity recognition first step is to collect the data and prepare a 
dataset which is then pre-processed and then the classification of data is carried out. This paper also presents various steps and techniques used in the process of recognition of human activities.

For the recognition of human activities from wearable, there are different classification techniques supervised and unsupervised (both) which are widely used by different researchers. Supervised techniques are considered more accurate when compared to unsupervised techniques [9].

IoT has numerous applications where different types of sensors are used in different types of applications. This paper studies different IoT applications and enlightens which smart application demands which type of sensor. Besides, various types of wearable sensors which are used for monitoring of human activities and application area where these are used are also presented in this paper.

This paper presents a detailed review of different locations where we can place wearable sensors and explains which one is ideal for a given group. It is also considered whether the sensor is comfortably wearable or not. For this, it should be small, lightweight, and able to provide the information content for which it is meant [10]. The positioning of wearable sensors along with the selection of relevant features for different activities pose challenges in this area. One of the limitations of wearable sensors in the prediction of optimal location on the body of the subject which can provide the most relevant features regarding activity classification [10].

Furthermore, various open areas are also explored in this paper so that a possible future research direction could be recognized and in the near future, we will come up with a good solution in this direction.

Section II of this paper presents various application areas of IoT where different types of sensors are used. Then, in section III, an analysis is made to identify the type of sensors that are used in different IoT applications. This helps various researchers in this area to identify which sensor is suited best for any specific type of application. Sensors are also used for HAR systems. Section IV presents several HAR systems and then categorizes various types of activities that are recognized in different HAR systems. Finally, a new HAR system is proposed in section V that helps the users to identify different activities. And soon, we come up with the detailed design of this HAR system that would be beneficial for the society in general.

\section{Sensor Applications for a Smarter World}

There are various issues and challenges in implementing and operating smart environment applications. Advanced smart applications need sustainability, reliability, and efficiency. A smart environment commonly has various kinds of Sensor nodes that control the current environment condition and energy usage. Sensors give their measurements and observations to the control system and then intelligent algorithms are implemented by the control system in the network. For surveillance and monitoring of smart environment applications, sensors are implemented in wide areas. Various examples are Highway Data Collection, Electric Toll Collection System, Traffic Signal Monitoring, Structural Health Management System, Traffic Management System, etc. Figure 1 presents the major application areas of IoT where IoT is marking its presence to create a smart environment.

In this section, we have presented some of the applications of IoT sensors.

\subsection{Intelligent Transportation:}

It is one of the prominent domains of Smart City application. All aspects of VANET safety involve communication, decision making, observation, and action. Currently, self-driving cars are also high attention to intelligent transportation. And this is possible due to sensors embedded in them [11].

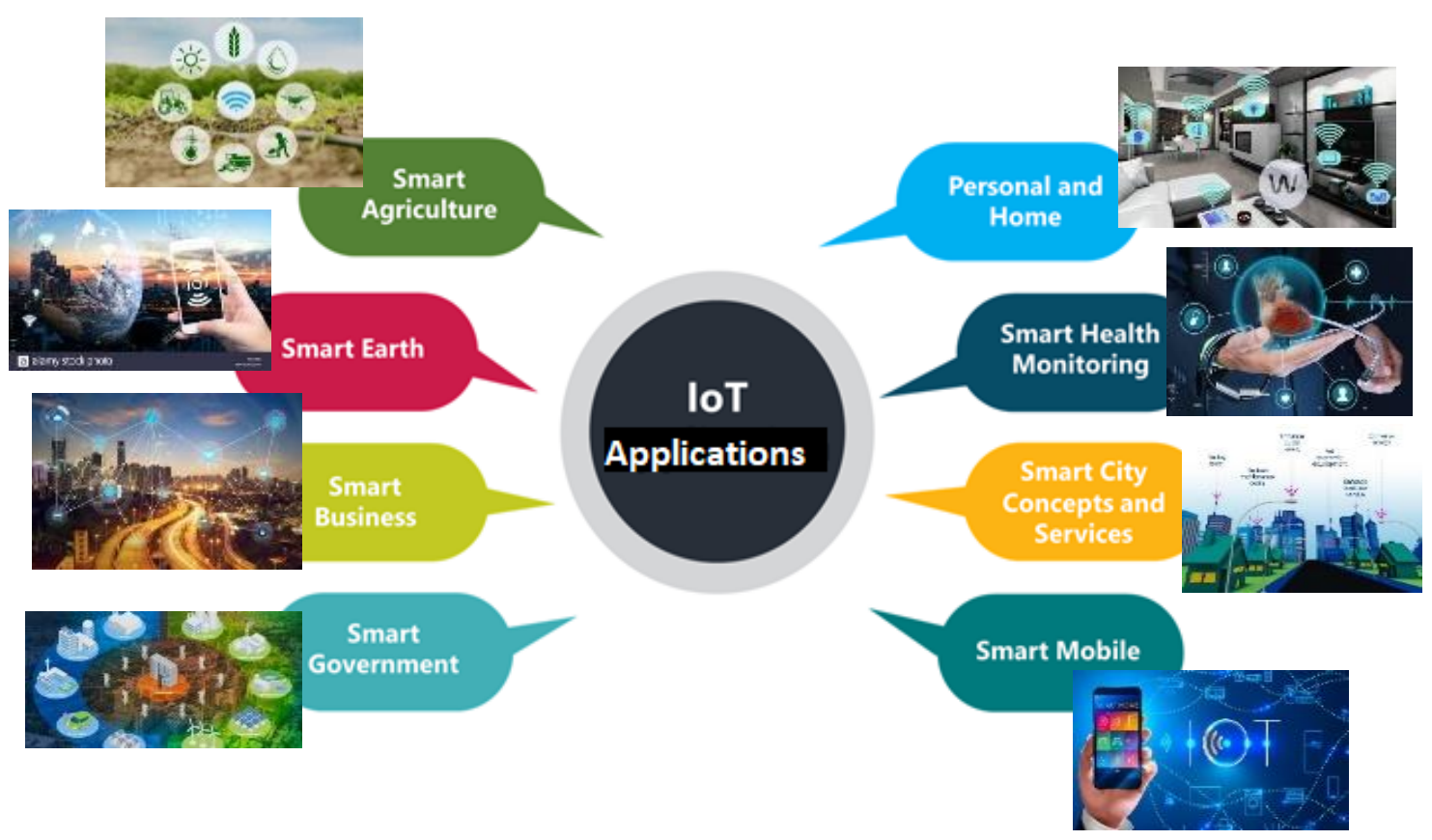

Figure 1: Major IoT Applications 


\subsection{Military Applications}

In Defense Operation, Information sharing between soldiers is an important issue for effective combat. But, in the hostile sector, there is a very difficult process for building infrastructure, most of which, communication infrastructure has always become the first target of the enemy. Hence creation and maintaining communication infrastructure is the essential issue of a military operation. In defense Operations, Information sharing between soldiers is an important issue for minimal casualties and effective combats. Sensors are used in various areas in military like Reconnaissance of opposing forces and terrain, Battlefield surveillance, Battle damage assessment, Equipment, and ammunition, monitoring friendly forces.

\subsection{Medical and Healthcare Applications}

In the medical \& Healthcare applications, the Sensors promises to solve various issues that are related to medical discoveries on incurable diseases. Most of the Healthcare devices are linked wirelessly through sensors [12]. In medical, IoT is used in identification management (IDM). In IDM each thing (device or user) needed to have a Unique Identification and it ensures identity and information safety. IDM also relays on sensors. Authors in [13], proposed a NIGHTCare platform that overnight detects the abnormal activities and events of patients in a health center. In this application, sensors are attached to the clothes of the patients. Another researcher in the same area presented a low-cost rural ehealth monitoring model [14]. A similar smart health application by authors in [15] monitors the physiological parameters of the patients. Sensors that detect the temperature and humidity of a patient's body are used by the researchers in [16]. These sensors are placed inside the shoe insole.

\subsection{Vehicular Network}

VANET usually linked to the internet using APs using WLAN (wireless local area network) Technology such as Sensors, Bluetooth, WiMAX, WI-Fi. Hence sensors play an important role in this area. VANET application: Prevention of Disaster scenarios, Evacuation emergency, Proximity, and correlation analysis, Traffic flows, Congestion, Mobile sensor networks through vehicle communications, Road congestion avoidance, etc.

\subsection{Smart Cities}

Sensors aim to make things convenient and dynamic. Things can exchange data using wireless technology and provides many convenient services. We need new techniques to address various challenges in Smart City. We need to come up with a new approach, where the internet of thing is used to minimize the communication problem of the sensors. IoT deployed in a smart environment comprises IoT devices to transmit the packets to the sink. The Internet of Things encapsulates sensors as an essential component. For surveillance and monitoring of smart environment applications, sensors are implemented in wide areas. Various examples are Highway Data Collection, Electric Toll Collection System, Traffic Signal Monitoring, Structural Health Management System, Traffic Management System, etc [11] [17].

\subsection{Heterogeneity of Network}

Intelligent transportation and smart building involve Sensors to add the different components within the network. In these systems, sensors need to be capable to co-exist. The network requires accurate mapping of different control information within the Network Stack.

\subsection{Wired and Wireless Connectivity}

Almost all the applications in the smart environment require wireless communication and wireless communication need sensors. The Internet of Things has a discrete topology that ties heterogeneous things/ devices together. Since in the Smart City, the data is inexhaustible and it has an expiration time associated with it means the information must reach the destination within a limited mention time and with good connectivity. Sensors play a vital role to provide good connectivity.

\subsection{Smart Environment/ Smart Earth}

Sensors are also used for monitoring the environmental conditions of the earth to provide a smart environment. It helps in the development of the country. This is not so simple and easy as it seems to be. Monitoring earth is a challenging area because of various constraints like harsh operating conditions and difficulty in maintenance. Also, highly developed and latest advancements make it more challenging [18].

\subsection{Smart Water}

Water is an essential part of life for every living being. It is necessary to monitor the quality of water in terms of pollution, minerals, its level from the ground, and many more. Sensors are widely used to monitor water quality. It's one widely used application is detecting leakages and water overflow in tanks. This not only saves water but also assist in water management [19]. Some of the smart applications of water are detecting water leakages, monitoring seawater pollution level, smart pools, monitoring river floods, dams and reservoirs levels, and many more. All this is possible due to the sensors that are attached to various sites.

\subsection{Smart Security:}

IoT is marking its presence in almost all areas. In this scenario, our day-to-day very personal data is over network all the time. So, it becomes more important to secure this data. Also, sensors are attached to the walls of the containers in various applications for security purposes like intrusion detection systems in homes and offices, monitoring gas levels in sensitive applications, smart monitoring radiation levels, and early detection of leakages. This not only saves a life but also helps in timely management. Sensors are also used for implementing smart perimeters around specific areas that restrict the entry of intruders at that place. A similar application is designed by the authors in [19] where an intelligent intrusion system attached at door, named ADXL 345 accelerometer is given. A similar embedded security framework for IoT is proposed by the researchers in [20].

\subsection{Smart Agriculture}

Sensors are extensively used in several agriculture applications. When attached to the soil, it detects moisture in the soil and by monitoring weather conditions like humidity and temperature it sprinkles water and pesticides in the fields according to the grown crops. An application model is proposed by the 
researchers in [21] which detects the humidity, moisture in the soil, rainfall, $\mathrm{pH}$ value of soil, temperature, and seed recognition. A similar study in [22] used a drone for smart agriculture. Water sprinkling in IoT agriculture is presented in [23], it is a case of vertical farming.

\subsection{Smart Homes}

Sensors are widely used in creating a smart home. Sensors are attached to various household devices like refrigerator, doors, lights, fan, AC, washing machine, grass, water tank, kids' room, school bags, curtains, tv, and almost to every device or appliance. Its application for smart home includes intruder detection, switching on/off electric appliances, opening/closing doors, sending meeting information, smart cameras for guests, detecting the amount of ingredient in cooking and so many. This all needs sending alerts to provide a better lifestyle. Various researchers worked in this area. Researchers in [24] proposed an intrusion detection system that intimates the owner via messages whenever senses motion in the door.

\section{Analysis of IoT Applications and Sensors}

Different types of sensors are widely used in different IoT applications. After analyzing different applications of IoT, this section recommends which sensors are more suitable and efficient for a particular application. For example, smart city monitors movement on highways which requires position sensor, velocity sensor, accelerometer sensor; toll collection system requires proximity sensor, position sensor, and accelerometer sensor; traffic management in a smart city requires infrared sensor, GPS, and position sensor; and to monitor building structural health, it requires humidity, pressure and temperature sensors.

When talking about a smart environment, it requires monitoring of weather conditions for which a light sensor, temperature sensor, humidity sensor, and gyroscope are required. Whereas, to monitor the emission of harmful gases in the environment, there is a need for chemical sensors, biosensors, and also optical sensors.

Similarly, smart agriculture requires monitoring of soil via humidity and chemical sensors. For monitoring weather conditions for sprinkling water, it requires temperature, humidity sensors. When there is a need for monitoring of intruders in the field it requires proximity or position sensor. A water quality sensor helps in detecting the water level of the soil.

Smart health monitors a patient, so it requires different biorelated sensors that detect abnormal behavior in a patient like biosensors, chemical sensors, magneto sensors, and pressure sensors. To detect the physical location of patients or health providers there is a need of a position sensor.

Different types of sensors are used and required in different IoT applications. Figure 2 shows which type of sensor is used for a particular IoT application.

The analysis of various IoT applications and sensors shows that some applications require more than one type of sensor. Besides, selecting any sensor for an IoT application requires analyzing and identifying different types of activities for which we need sensors.

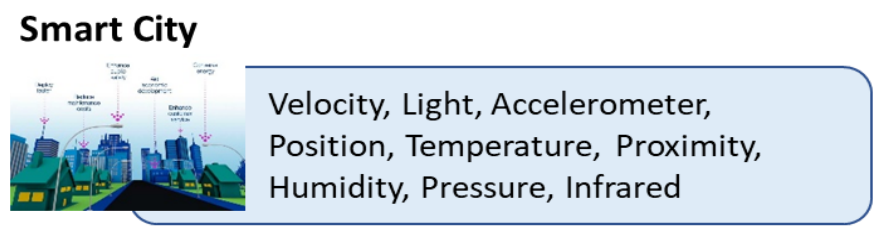

Smart Environment

Light, Temperature, Humidity,
Chemical, Gyroscope, Bio Sensors,
Chemicals, Accelerometer, Optical

$\begin{array}{ll}\text { Smart Water } & \\ & \begin{array}{l}\text { Temperature, Humidity, Occupancy, } \\ \text { Water Quality }\end{array}\end{array}$

Smart Building

Smart Health

Chemicals, Magneto, Accelerometer,
Pressure

Smart Home

Light, Gyroscope, Biosensors,
Chemicals, Magneto, Accelerometer,
Temperature, Proximity, Position,
Infrared

Smart Security

Light, Gyroscope, Chemical, Magneto,
Accelerometer, Temperature, Infrared

Smart Retail

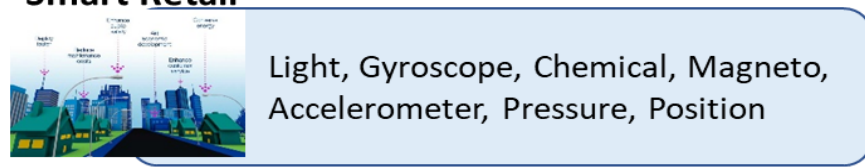

Smart Transport

Gyroscope, Pressure, chemicals,
Magneto, Accelerometer,
Temperature, Motion, Infrared

Figure 2: Types of sensors used in major IoT applications

\section{Human Activity Recognition (HAR) System}

HAR System detects human activities by observing various parameters through different sensors attached to the human body via different wearables. Various researchers worked in this field and proposed numerous HAR systems. 


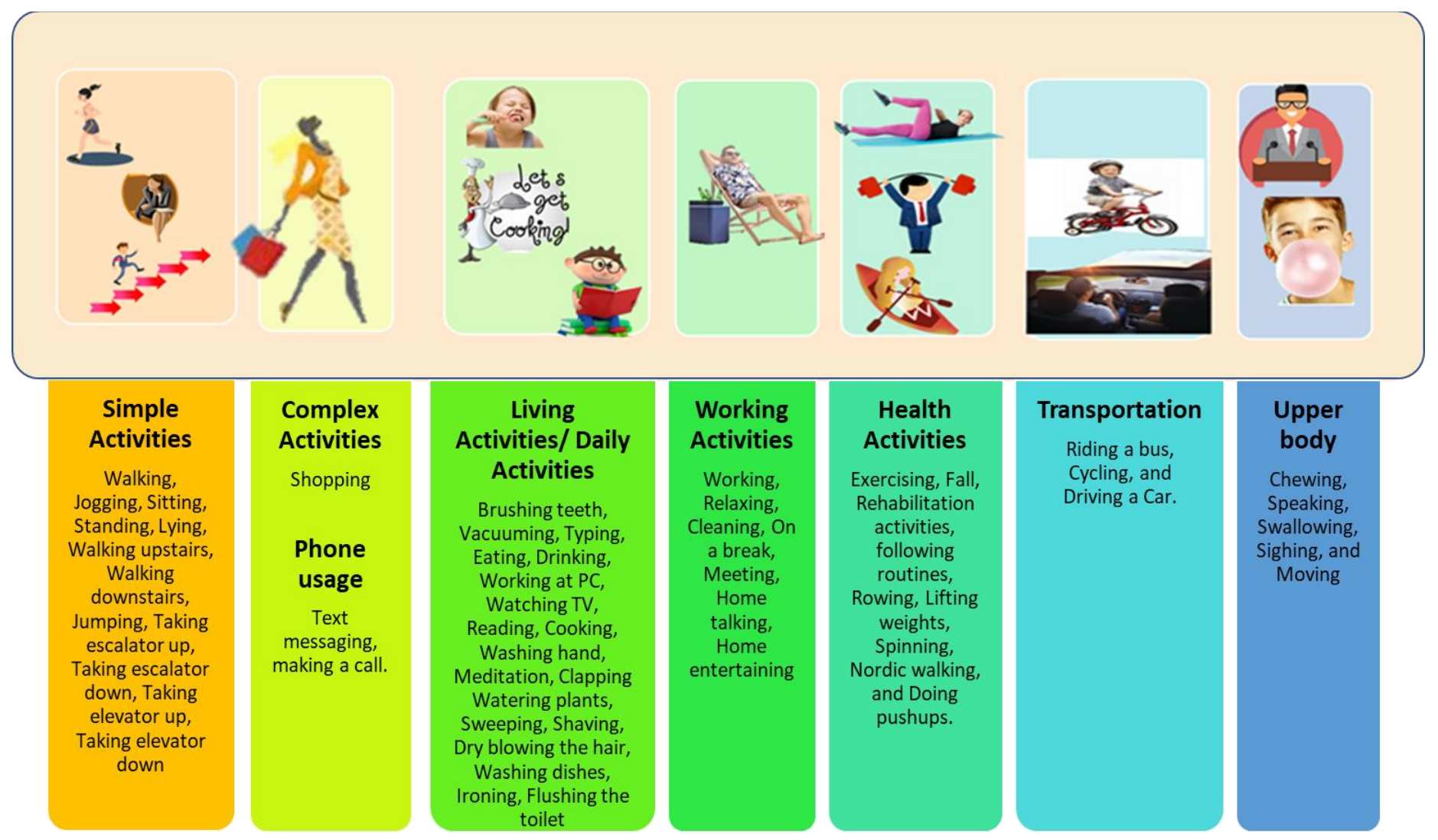

Figure 3: Various types of activities according to category

These monitoring systems are usually smaller and are wearable. Various researchers worked on detecting human activities by observing different parameters. It can be used for the monitoring of people of any age group like for infants, young people, disabled people, and elderly people in a solitary life. Researchers in [25] presented a wearable monitoring system for a patient that monitors various physiological parameters of the patient in healthcare. Notifications are sent to the concerned (doctor/ relative) in any abnormal event via a twitter account. Another similar study in [26] proposed a personalized health monitoring system. This system attaches a sensor inside the shoe that monitors the pressure, temperature, and humidity and sends the health data to the user via a hand-held device. A smart university campus is proposed by the authors in [27] to make smart rooms, smart parking, and smart education a reality. Wearable sensors are mostly used to detect physical movements but these can also be used for monitoring academic performance, analyzing sleep quality/patterns, stress level, and mental health conditions. Researchers in [8] gathered data from 66 individuals and analyzed behavioral and physiological patterns. They also identified factors affecting performance in academics, sleep quality, stress level, and mental health. HAR systems recognize various activities these are generally classified in different groups. Figure 3 enlists these types of activities according to the category.

\section{Security Mechanism in IoT}

As we know that in IoT enabled smart environments, everything is connected via the internet and data is communicated with other similar devices. In IoT based environment, there are approximately 6 times IoT enabled devices than people which are connected in IoT. Every minute, every second our very personal and sensitive data is over the network. This is serious and couldn't be overlooked. So, this smart environment requires an even more strong security mechanism than ever before. To fulfil the needs of IoT security, strong cryptographic solutions are required. Numerous security algorithms are present now-a-days like AES, DES, RSA, and so many. But using these traditional security algorithms in the IoT environment is not possible because of various constraints. As sensors are the main elements of IoT which are attached to even very small devices. So, implementing traditional security mechanisms in IoT enabled devices is not possible as these are having high memory requirements that are not available in small sensors. Also, there is a different operating environment, low available power, and frequent maintenance in IoT enabled devices is not possible. So, there is a need for such a security mechanism which fulfils the needs of constraint devices. In this direction, there comes a new area of cryptography, which is known as lightweight cryptography. Numerous researchers in the area of IoT security has given different lightweight security algorithms. Moving in the same direction, we have also proposed three different lightweight security algorithms, BRIGHT [28], [29], [30], and UBRIGHT [31]. A total of 9 different security algorithms are proposed in these three security algorithms.

\section{Proposed Work}

In this section, a HAR System is proposed. This system enables users to identify various types of activities. Security is an essential part of any HAR system, so in the proposed system for enhancing the security, one of the newly designed lightweight cipher out of BRIGHT, UBRIGHT, and Expanded-BRIGHT is proposed to be used. 

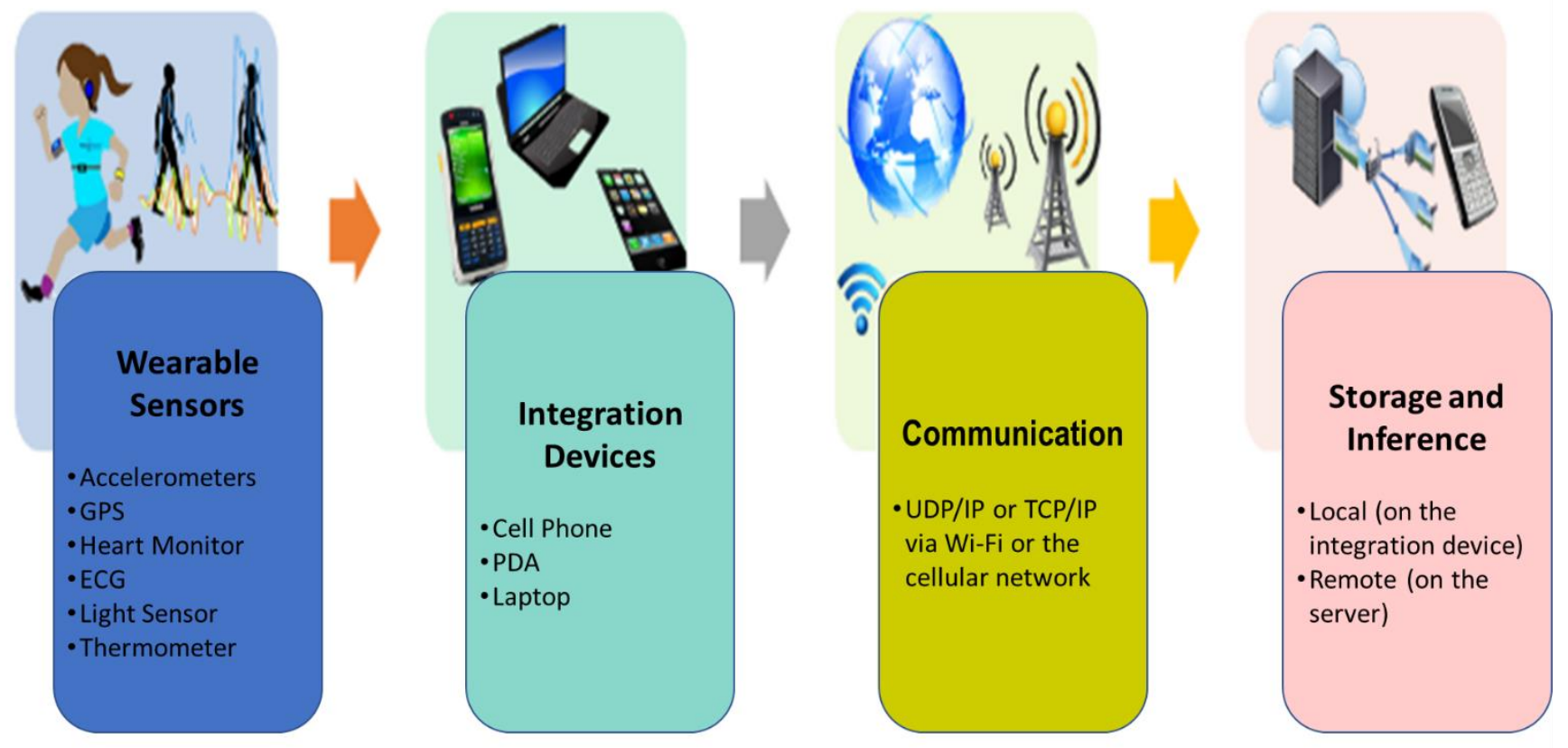

Figure 4: Generic data acquisition architecture for Proposed HAR systems

The first step in any HAR system is collecting raw data using sensors which are then passed through feature extraction and learning processes that recognize the activities. This is achieved through machine learning techniques that may be supervised or unsupervised or semi-supervised.

Figure 4 represents generic data acquisition architecture for the proposed HAR systems. First using different wearable sensors or accelerometer, data are gathered which is sent to some integrated devices where processing is carried out.

Feature Extraction is the next step where the sensors sampling rate is faster as compared to the time taken by human activities. A single sample at any specific instant of time is not sufficient to provide useful information. So, to successfully recognize any activity sampling must be considered for time windows rather than at any specific instant of time. The main underlying problem is that it is not possible to make a comparison between two-time windows. It's almost impossible for signals from the same subject performing the same activity to be identical. So, feature extraction comes into the picture and plays an important role in any HAR systems. There are two types of techniques for feature extraction from time-series data, these are statistical and structural. To extract features from the raw data, statistical methods make use of quantitative characteristics of the data. Fourier transform and the wavelet transform are examples of statistical methods. The methods in which interrelationship among data are considered into account are known as structural approaches.

Learning is a necessary step to build useful patterns that describe and analyze the data. Machine learning is carried out from examples or observations called a training set. Machine learning may be supervised or unsupervised. In unsupervised learning, it is quite not possible to distinguish the activities that are to be recognized. In supervised approaches, the labeling of different activities along with supervising collection process is carried out by some person whereas in an unsupervised learning technique no person is involved. Different researchers in this area are working on both supervised and unsupervised learning techniques. Then this data is communicated to the concerned and finally stored on some integration device or remote server.

\section{Conclusion}

IoT has numerous applications where different types of sensors are used in different types of applications. This paper discusses different IoT applications and enlightens which smart application demands which type of sensor. Besides, various types of wearable sensors which are used for monitoring of human activities and application area where these are used are also presented in this paper. The positioning of wearable sensors along with the selection of relevant features for different activities pose challenges in this area. Our very personal and sensitive data is over the network in this smart environment. This is serious and couldn't be ignored. So, it requires a strong security mechanism in the form of lightweight cryptography. In this paper, a HAR system is also proposed that enables users to identify various types of activities. To provide high enough security to the proposed system, very efficient and secure crypto solutions are also used.

\section{References}

[1] D. Sehrawat, N. S. Gill, "Smart Sensors: Analysis of Different Types of IoT Sensors" in 2019 3rd International Conference on Trends in Electronics and Informatics (ICOEI) (523-528), 2019. IEEE. https://doi.org/10.1109/ICOEI.2019.8862778

[2] D. Sehrawat, N. S. Gill, "Security Requirements of IoT Applications in Smart Environment", in: Proc. of 2nd International Conference on Trends in Electronics and Informatics (ICOEI 2018), 324-329, 2018. https://doi.org/10.1109/ICOEI.2018.8553681

[3] M. Ambrosin, A. Anzanpour, M. Conti, T. Dargahi, S. R. Moosavi, A. M. Rahmani, P. Liljeberg, "On the Feasibility of Attribute-Based Encryption on Internet of Things Devices", IEEE Micro, 36(6), 25-35, 2016. http://doi.org/10.1109/MM.2016.101

[4] D. Sehrawat, N. S. Gill, "Deployment of IoT based Smart Environment: Key Issues and Challenges", International Journal of Engineering \& Technology. 7(2), 544-550, 2018. http://dx.doi.org/10.14419/ijet.v7i2.9504

[5] H. Lee, K. Kim, J. Kwon, "A pervasive interconnection technique for efficient information sharing in social iot environment," Int. J. Smart Home, 10(1), 9-22, 2016

[6] S. Kale, M. Satendra, P. Patil, "IOT based Wearable Biomedical Monitoring System," in International Conference on Trends in Electronics and 


\section{Sehrawat et al. / Advances in Science, Technology and Engineering Systems Journal Vol. 5, No. 4, 516-522 (2020)}

Informatics ICEI 2017 IOT, 2017, 971-976. https://doi.org/10.1109/ICOEI.2017.8300852

[7] J. Wilden, A. Chandrakar, A. Ashok, N. Prasad, "IoT Based Wearable Smart Insole," in Wireless Summit (GWS), 2017 Global IEEE., 2017, 186-192. https://doi.org/10.1109/GWS.2017.8300466

[8] A. Sano, A. J. Phillips, Z. Y. Amy, A. W. McHill, S. Taylor, N. Jaques, N., ... \& R. W. Picard, "Recognizing academic performance, sleep quality, stress level, and mental health using personality traits, wearable sensors and mobile phones", in 2015 IEEE 12th International Conference on Wearable and Implantable Body Sensor Networks (BSN) (1-6) ， 2015. IEEE. https://doi.org/10.1109/BSN.2015.7299420

[9] F. Attal, S. Mohammed, M. Dedabrishvili, F. Chamroukhi, L. Oukhellou, Y. Amirat, "Physical human activity recognition using wearable sensors" Sensors, 15(12), 31314-31338, 2015. https://doi.org/10.3390/s151229858

[10] L. Atallah, B. Lo, R. King, G. Z. Yang, "Sensor placement for activity detection using wearable accelerometers" in 2010 International conference on body sensor networks, 24-29, 2010. IEEE. https://doi.org/10.1109/BSN.2010.23

[11] A. Zanella, N. Bui, a Castellani, L. Vangelista, M. Zorzi, "Internet of Things for Smart Cities," IEEE Internet Things, 1(1), 22-32, 2014. 10.1109/JIOT.2014.2306328

[12] D. Zissis, D. Lekkas, "Addressing cloud computing security issues", Futur. Gener. Comput. Syst., 28(3), 583-592, 2012. Available Online: https://doi.org/10.1016/i.future.2010.12.006

[13] C. Occhiuzzi, C. Vallese, S. Amendola, S. Manzari, G. Marrocco, "NIGHTcare: A passive RFID system for remote monitoring and control of overnight living environment", Procedia Comput. Sci., 32, 190-197, 2014. https://doi.org/10.1016/j.procs.2014.05.414

[14] R. Chanchal, J. Chaman, W. Arif, "HEMAN : Health Monitoring and Nous", in IEEE WiSPNET 2017 conference., 2017, 2115-2119. 10.1109/WiSPNET.2017.8300134

[15] S. Kale, M. Satendra, P. Patil, "IOT based Wearable Biomedical Monitoring System", in International Conference on Trends in Electronics and Informatics ICEI 2017 IOT, 2017, 971-976. 10.1109/ICOEI.2017.8300852

[16] J. Wilden, A. Chandrakar, A. Ashok, N. Prasad, "IoT Based Wearable Smart Insole", in: Wireless Summit (GWS), 2017 Global IEEE., 2017, 186-192. 10.1109/GWS.2017.8300466

[17] P. Patil, "Smart IoT Based System For Vehicle Noise And Pollution Monitoring Piyush," in Proc. of International Conference on Trends in Electronics and Informatics, 2017, 322-326. 1109/ICOEI.2017.8300941

[18] M. T. Lazarescu, "Design of a WSN Platform for Long-Term Environmental Monitoring for IoT Applications," IEEE J. Emerg. Sel. Top. CIRCUITS Syst., 3, 45-54, 2013. 10.1109/JETCAS.2013.2243032

[19] R. Khan, S. U. Khan, R. Zaheer, S. Khan, "Future internet: The internet of things architecture, possible applications and key challenges," in: Proc. of 10th Int. Conf. Front. Inf. Technol. FIT 2012, April 2017, 257260. 10.1109/FIT.2012.53

[20] A. Stango, N. R. Prasad, J. Sen, "Proposed Embedded Security Framework for Internet of Things ( IoT )," in: Proc. of 2nd International Conference on Wireless Communication, Vehicular Technology, Information Theory and Aerospace \& Electronic Systems Technology (Wireless VITAE), IEEE, 2011, 1-5. 10.1109/WIRELESSVITAE.2011.5940923

[21] P. Patil, V. Sachapara, "Providing Smart Agricultural Solutions/Techniques By Using Iot Based Toolkit," in: Proc. of International Conference on Trends in Electronics and Informatics ICEI 2017 Providing, 2017, 327-331. 10.1109/ICOEI.2017.8300942

[22] A. K. Saha et al., "IOT-Based Drone for Improvement of Crop Quality in Agricultural Field," IEEE Internet Things J., 612-615, 2018. 10.1109/CCWC.2018.8301662

[23] M. Ikhwan, N. M. Thamrin, "IoT Implementation for Indoor Vertical Farming Watering System", IEEE Internet Things J., 89- 94, 2017. 10.1109/ICEESE.2017.8298388

[24] S. Nazeem Basha, S. Jilani, M. Arun, "An Intelligent Door System using Raspberry Pi and Amazon Web Services IoT", Int. J. Eng. Trends Technol., 33(2), 84-89, 2016.

[25] M. Ermes, J. Pärkkä, J. Mäntyjärvi, L. Korhonen, "Detection of daily activities and sports with wearable sensors in controlled and uncontrolled conditions", IEEE transactions on information technology in biomedicine, 12(1), 20-26, 2008. https://doi.org/10.1109/TITB.2007.899496

[26] L. Bao, S. S. Intille, "Activity recognition from user-annotated acceleration data", in International conference on pervasive computing, 1-17, 2004. Springer, Berlin, Heidelberg. https://doi.org/10.1007/978-3-540-24646-6_1

[27] K. Kunze, P. Lukowicz, H. Junker, G. Tröster, "Where am i: Recognizing on-body positions of wearable sensors", in International Symposium on
Location-and Context-Awareness, 264-275, 2005. Springer, Berlin, Heidelberg. https://doi.org/10.1007/11426646_25

[28] D. Sehrawat, N. S. Gill, "BRIGHT - Proposed Family of Lightweight Block Ciphers for IoT-Enabled Smart Environment", International Journal of Innovative Technology and Exploring Engineering (IJITEE). 8(9), 584-592, 2019.https://www.ijitee.org/wpcontent/uploads/papers/v8i9/I7778078919.pdf

[29] D. Sehrawat, N. S. Gill, "BRIGHT: A Small and Fast Lightweight Block Cipher for 32-bit Processor", International Journal of Engineering and Advanced Technology, 8(5), 1549-556, 2019. https://www.ijeat.org/wpcontent/uploads/papers/v8i5/E7302068519.pdf

[30] D. Sehrawat, N. S. Gill, "Performance Evaluation of Newly Proposed Lightweight Cipher, BRIGHT", International Journal of Intelligent $\begin{array}{lllll}\text { Engineering \& Systems, } & \mathbf{1 2}(4), & 71-80, & 2019\end{array}$ http://www.inass.org/2019/2019083108.pdf

[31] D. Sehrawat, N. S. Gill, "UBRIGHT: Ultra BRIGHT, a Tiny and Fast Ultra Lightweight Block Cipher for IoT", International Journal of Scientific \& Technology Research, 9(2), 1063-1068, 2020. http://www.ijstr.org/finalprint/feb2020/Ultra-Bright-A-Tiny-And-Fast-Ultra-Lightweight-BlockCipher-For-Iot.pdf 